\title{
Equivalent electron densities at reflection heights of tweek atmospherics in the low-middle latitude D-region ionosphere
}

\author{
H. Ohya ${ }^{1}$, M. Nishino ${ }^{2}$, Y. Murayama ${ }^{3}$, and K. Igarashi ${ }^{3}$ \\ ${ }^{1}$ Department of Electronics and Mechanical Engineering, Chiba University, Chiba 263-8522, Japan \\ ${ }^{2}$ Solar-Terrestrial Environment Laboratory, Nagoya University, Toyokawa 442-8507, Japan \\ ${ }^{3}$ Communications Research Laboratory, Koganei, Tokyo 184-8759, Japan
}

(Received May 1, 2003; Revised November 7, 2003; Accepted November 7, 2003)

\begin{abstract}
Tweek atmospherics are ELF/VLF pulse signals with frequency dispersion characteristics that originate from lightning discharges and propagate in the Earth-ionosphere waveguide mode over long distances. In this paper, we estimate equivalent nighttime electron densities at reflection heights in D-region ionosphere at low-middle latitudes by accurately reading the first-order mode cut-off frequency of tweek atmospherics. The estimation method was applied to tweek atmospherics received simultaneously at Moshiri and Kagoshima in Japan. Equivalent electron densities ranged from $20-28 \mathrm{el} / \mathrm{cm}^{3}$ at ionospheric reflection heights of $80-85 \mathrm{~km}$. Comparing our estimates with electron density profiles obtained from the IRI-95 model, MF radar measurements, and rocket experiments revealed almost consistent results for the lower part of the D-region ionosphere. The tweek method has the unique advantage of enabling reflection-height (equivalent electron densities) monitoring over a wide area of several thousand kilometers.
\end{abstract}

Key words: Tweek atmospherics, cut-off frequency, equivalent electron densities (reflection heights).

\section{Introduction}

The altitude of the D-region ionosphere is too low for satellite measurements of electron density and too high for balloon measurements; therefore several methods have been tried. Rockets have been used (e.g., Maeda, 1971; Nagano and Okada, 2000), but rocket experiments are restricted by the timing of flights. Ground-based active experiments using ionosondes and incoherent scatter radars in the HF-VHF range can be conducted at any time, but these methods cannot receive ionospheric echoes due to the low electron densities $\left(<10^{3} \mathrm{el} . / \mathrm{cm}^{3}\right)$ in the D-region ionosphere. MF radar has been used (e.g., Igarashi et al., 2000), but this method is hindered by the high costs of regular operations for active radio measurements. Reception of radio-wave signals propagated from VLF/LF transmitters has also been adopted (Thomas and Harrison, 1970; Bickel et al., 1970; Thomson, 1993), but the region available for electron density measurements is restricted to the propagation path between the transmitter and the receiver.

In this study, we used tweek atmospherics to estimate electron densities in the D-region ionosphere. Tweek atmospherics are ELF/VLF pulse signals with certain frequency dispersion characteristics. Tweek atmospherics originate from lightning discharges and are propagated in the Earthionosphere waveguide mode over long distances. Previous studies of tweek atmospherics have focused on theoretical considerations related to waveform analysis and on propagation mechanism (Outsu, 1960; Yamashita, 1978; Yano et al., 1989; Ryabov, 1992; Yedemsky et al., 1992). Recently,

Copy right(C) The Society of Geomagnetism and Earth, Planetary and Space Sciences (SGEPSS); The Seismological Society of Japan; The Volcanological Society of Japan; The Geodetic Society of Japan; The Japanese Society for Planetary Sciences. studies have advanced to elucidate detailed wave characteristics through the measurement of tweek arrival directions (Hayakawa et al., 1994).

Figure 1 shows an example of a frequency-time diagram of nighttime ELF/VLF wave signals received at Moshiri $\left(44.37^{\circ} \mathrm{N}, 142.27^{\circ} \mathrm{E}\right)$. The diagram of strong tweek atmospherics at about $16.1 \mathrm{~s}$ shows the frequency dispersion at cut-off frequencies near $1.8 \mathrm{kHz}$ and $3.6 \mathrm{kHz}$. Frequency dispersion is caused by the wave group velocity becoming very small near the cut-off frequencies due to multiple reflections between the D-region ionosphere and the Earth's surface. Generally, more frequent tweek atmospherics are received at night, when attenuation is less. On average, 100 200 tweek atmospherics are received per minute at night. Here, we focus on the characteristics of the frequency dispersion of tweek atmospherics. These characteristics provide information about ionospheric reflection heights (equivalent electron densities) and propagation distances in the Earthionosphere waveguide.

First, we calculated reflection heights by accurately reading the first-order mode cut-off frequency of the tweek atmospherics. From this reading, we estimated equivalent electron densities at the reflection heights. Next, we evaluated the estimated electron densities and compared these densities to the electron density profiles given by the International Reference Ionosphere (IRI) model, simultaneous MF radar measurements, and previous rocket experiments.

\section{Theoretical Background of Tweek Atmospherics} The refractive index of wave propagation in magnetoactive plasma is expressed by the famous Appleton-Hartree formula (e.g., see Budden, 1961): 

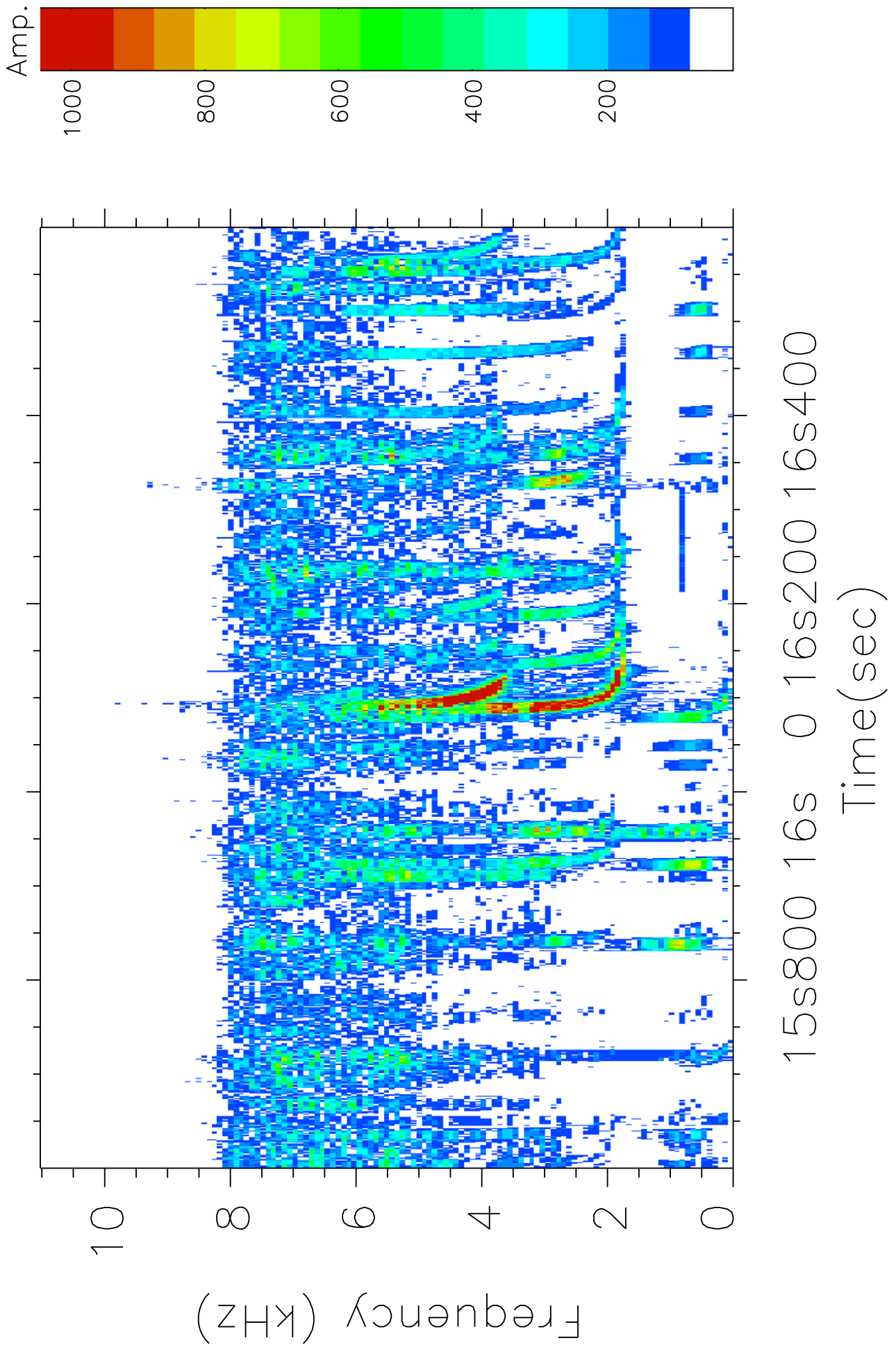

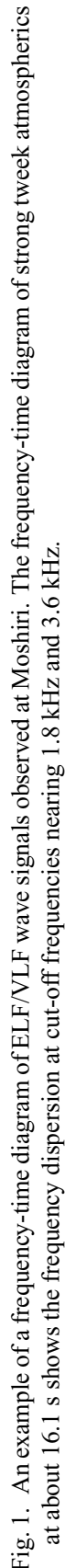




$$
\begin{aligned}
n_{r}^{2} & =1 \\
& -\frac{2 X(1-X-j Z)}{2(1-j Z)(1-X-j Z)-Y_{T}^{2} \pm \sqrt{4 Y_{L}^{2}(1-X-j Z)^{2}+Y_{T}^{4}}},(1)
\end{aligned}
$$

where

$$
\begin{gathered}
X=\left(\frac{\omega_{p}}{\omega}\right)^{2} \\
Y=\frac{\omega_{H}}{\omega} \\
Z=\frac{v}{\omega} \\
e Y_{L}=Y \cos \alpha \\
Y_{T}=Y \sin \alpha
\end{gathered}
$$

and $n_{r}$ is the refractive index, $\omega$ is the angular frequency of the wave, $v$ is the angular collision frequency of electrons with neutrals, and $\alpha$ is the angle between the propagation direction of the wave and the external magnetic field vector.

The angular plasma frequency $\omega_{p}\left(=2 \pi f_{p}\right)$ is given by the following plasma frequency,

$$
f_{p}=\frac{1}{2 \pi} \sqrt{\frac{n_{e} e^{2}}{m \epsilon_{0}}} \approx 9.0 \sqrt{n_{e}},
$$

and the angular electron gyro-frequency $\omega_{H}$ is given as follows:

$$
\omega_{H}=\frac{e \mu_{0} H}{m},
$$

where $n_{e}$ is the electron density per $\mathrm{cm}^{3}, e\left(=-1.602 \times 10^{-19}\right.$ C) is an electric charge, $\epsilon_{0}\left(=8.854 \times 10^{-12} \mathrm{~F} / \mathrm{m}\right)$ is permittivity in a vacuum, $m\left(=9.109 \times 10^{-31} \mathrm{~kg}\right)$ is the mass of an electron, $\mu_{0}\left(=4 \pi \times 10^{-7} \mathrm{H} / \mathrm{m}\right)$ is permeability in a vacuum, and $H$ is the geomagnetic field strength. In Eq. (1), the upper sign " + " in the denominator corresponds to ordinarymode (O-mode) waves, and the lower sign "-" corresponds to extraordinary-mode (X-mode) waves.

Using quasi-longitudinal approximation, Yedemsky et al. (1992) and Hayakawa et al. (1994) demonstrated the possibility of full reflection for extraordinary waves in the lower ionosphere. O-mode waves can propagate into the ionosphere. In contrast, as the square of the refractive index $\left(n_{r}^{2}\right)$ for $\mathrm{X}$-mode waves decreases with an increase in electron density (with altitude), X-mode waves undergo full reflection at the altitude at which the refraction index $n_{r}$ becomes zero. Thus, X-mode waves cannot propagate into the ionosphere. However, quasi-longitudinal approximation is not needed for this study, because the full reflection condition can be derived directly from the Appleton-Hartree equation (Shvets and Hayakawa, 1998).

The $X$ value where $n_{r}^{2}$ becomes zero is given by the following equation, which shows wave cut-off without regard to propagation direction:

$$
\begin{gathered}
X=1 \\
X=1 \pm Y .
\end{gathered}
$$

Equations (4) and (5) represent O-mode and X-mode waves, respectively. The $\mathrm{X}$-mode waves correspond to $X=1+Y$ when $Y>1\left(\omega_{H}>\omega\right)$, and $X=1-Y$ when $Y<1$
$\left(\omega_{H}<\omega\right)$. Therefore, only $X=1+Y$ is used for ELF/VLF waves.

Thus, the electron density $n_{e}$ at the tweek atmospherics' reflection height is derived from $X=1+Y$ as follows:

$$
n_{e}\left(/ \mathrm{cm}^{3}\right)=1.241 \times 10^{-8} f_{p}\left(f_{p}+f_{H}\right) .
$$

$f_{p}\left(=\omega_{p} / 2 \pi\right)$ corresponds to the cut-off frequency of the tweek atmospherics for the first-order mode, and is replaced by the cut-off frequency $\left(f_{c}\right)$ in the following equation. Since we receive tweek atmospherics from lightning discharges occurred mainly in low-latitude and equatorial regions we take $f_{H}=1.1 \pm 0.2 \mathrm{MHz}$ according to the International Geomagnetic Reference Field (IGRF) model. The first-order mode cut-off frequency of tweek atmospherics usually ranges in $1.5-2.5 \mathrm{kHz}$. Then, $f_{H} \gg f_{p}$ is satisfied, resulting in that the Eq. (6) is replaced as follows:

$$
n_{e}\left(/ \mathrm{cm}^{3}\right)=1.241 \times 10^{-8} f_{c} f_{H} .
$$

As the result, electron densities are estimated as $n_{e}=27 \pm 5$ el. $/ \mathrm{cm}^{3}$ for the change of $f_{H}$, if we take $f_{c}=2.0 \mathrm{kHz}$ and $f_{H}=1.1 \mathrm{MHz}$. Thus, it is indicated that equivalent electron densities can be accurately estimated by the tweek method, in spite of the long propagation paths.

\section{Estimation of Reflection Heights and Source Po- sitions}

Magnetic tapes were used to record ELF/VLF signals of tweek atmospherics at Moshiri $\left(44.37^{\circ} \mathrm{N}, 142.27^{\circ} \mathrm{E}\right)$ and Kagoshima $\left(31.48^{\circ} \mathrm{N}, 130.72^{\circ} \mathrm{E}\right)$ located at middle and low latitudes, respectively. Figure 2 shows the locations of the two stations where observations were routinely carried out for two minutes (at 50-52 min) every hour. The two minutes of data were regarded as representative of each hour. The stronger nighttime tweek atmospherics were used for this study in order to accurately analyze tweek frequency dispersions. Analog ELF/VLF signals were digitized with a 16-bit A/D converter with a $16.00-\mathrm{kHz}$ sampling frequency.

The frequency dispersion of tweek atmospherics assumes a flat Earth-ionosphere waveguide mode. The modal equation is as follows:

$$
R_{g}(C) R_{i}(C) \exp (-i 2 k h C)=\exp (-i 2 \pi n)
$$

where $R_{g}(C)$ and $R_{i}(C)$ are the reflection coefficients at the ground and the ionosphere, respectively (Wait, 1970), and $k$ is the wave number, $h$ is the reflection height up to the bottom of the ionosphere, $C\left(=\sqrt{1-S^{2}}\right)$ is the cosine of the incident angle, and $n$ is the mode number. In general, $C$ and $S$ are complex numbers. The real part of $C$ is given as $\Re(C)=\lambda / 2 h=\pi /(k h)$, where $\lambda(=2 \pi / k)$ is the wave length. The phase velocity $v_{p}$ is given by

$$
v_{p}=\frac{\omega}{\Re(k S)},
$$

where $\mathfrak{R}(S)$ is the real part of $S$. The group velocity $v_{g}$ is then given by

$$
v_{g}=\frac{\partial \omega}{\partial\{\Re(k S)\}}=c \sqrt{1-\left(\frac{\pi}{k h}\right)^{2}},
$$




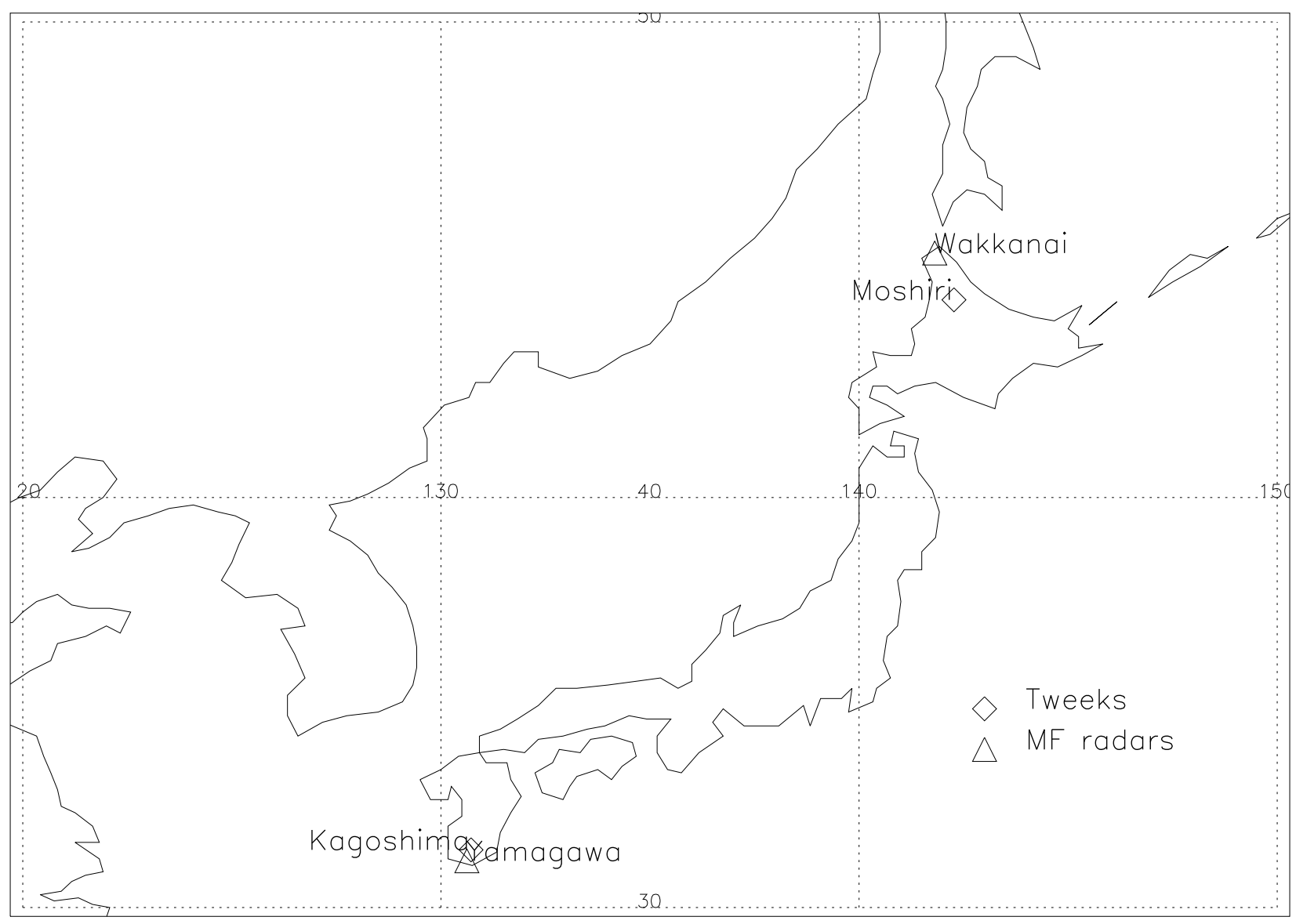

Fig. 2. ELF/VLF wave signal observation stations (Moshiri and Kagoshima) and the two stations (Wakkanai and Yamagawa, Japan) providing MF radar.

where $c$ is the light velocity (Shimakura et al., 1990). Thus, the propagation time $t_{g}$ is given as follows:

$$
t_{g}=\frac{d}{v_{g}}=\frac{d}{c \sqrt{1-\left(\frac{\pi}{k h}\right)^{2}}},
$$

where $d$ is the horizontal propagation distance. The reflection height $h$ is obtained by the first-order mode cut-off frequency $f_{c}$ as follows:

$$
h=\frac{c}{2 f_{c}},
$$

indicating that the first-order cut-off frequency is determined by $2 h$. In this case, the incident angle of tweek atmospherics to the reflection surface is nearly zero on the first-order mode cut-off frequency (Hayakawa et al., 1994). Consequently, the frequency dispersion of tweek atmospherics is given by the frequency-time domain $f\left(t_{g}\right)$, as follows:

$$
f\left(t_{g}\right)=\frac{f_{c} t_{g}}{\sqrt{t_{g}^{2}-\left(\frac{d}{c}\right)^{2}}} .
$$

Substituting $t_{g}=t-t_{0}$ for Eq. (13), where $t_{0}$ is the occurrence time of a lightning discharge, the frequency dispersion is given as

$$
f\left(t-t_{0}\right)=\frac{f_{c}\left(t-t_{0}\right)}{\sqrt{\left(t-t_{0}\right)^{2}-\left(\frac{d}{c}\right)^{2}}} .
$$

We fit the dynamic spectrum of tweek signals to Eq. (14) using a computer-calculated least-mean-square method and determined the parameters $f_{c}, t_{0}$ and $d$. Cut-off frequencies were determined for strong tweek signals that exceeded 20 $\mathrm{dB}$ from the background noise.

In the following section, electron density is equivalently calculated from Eq. (7) on the first-order mode cut-off frequency. Thus, the tweek method represents equivalent electron density at the reflection height over the entire propagation path of tweek atmospherics.

From the fitting process, we first obtained average values and standard deviations. We then excluded parameters if their standard deviations exceeded three times the average. By this method, $2-3 \%$ of the entire data set was excluded. As a result, reading errors of the cut-off frequency were about $\pm 38 \mathrm{~Hz}$, which corresponds to errors of $<1 \mathrm{el} . / \mathrm{cm}^{3}$ for the electron densities, $\pm 1.9 \mathrm{~km}$ for reflection heights between 70 and $80 \mathrm{~km}$, and $\pm 945 \mathrm{~km}$ for the propagation distance. Source positions of tweek atmospherics were fixed by the intersection of two circles drawn by the propagation distances from Moshiri and Kagoshima. Thus, the source position error is within $\pm 8.51^{\circ}$ within the geographical coordinate system. 

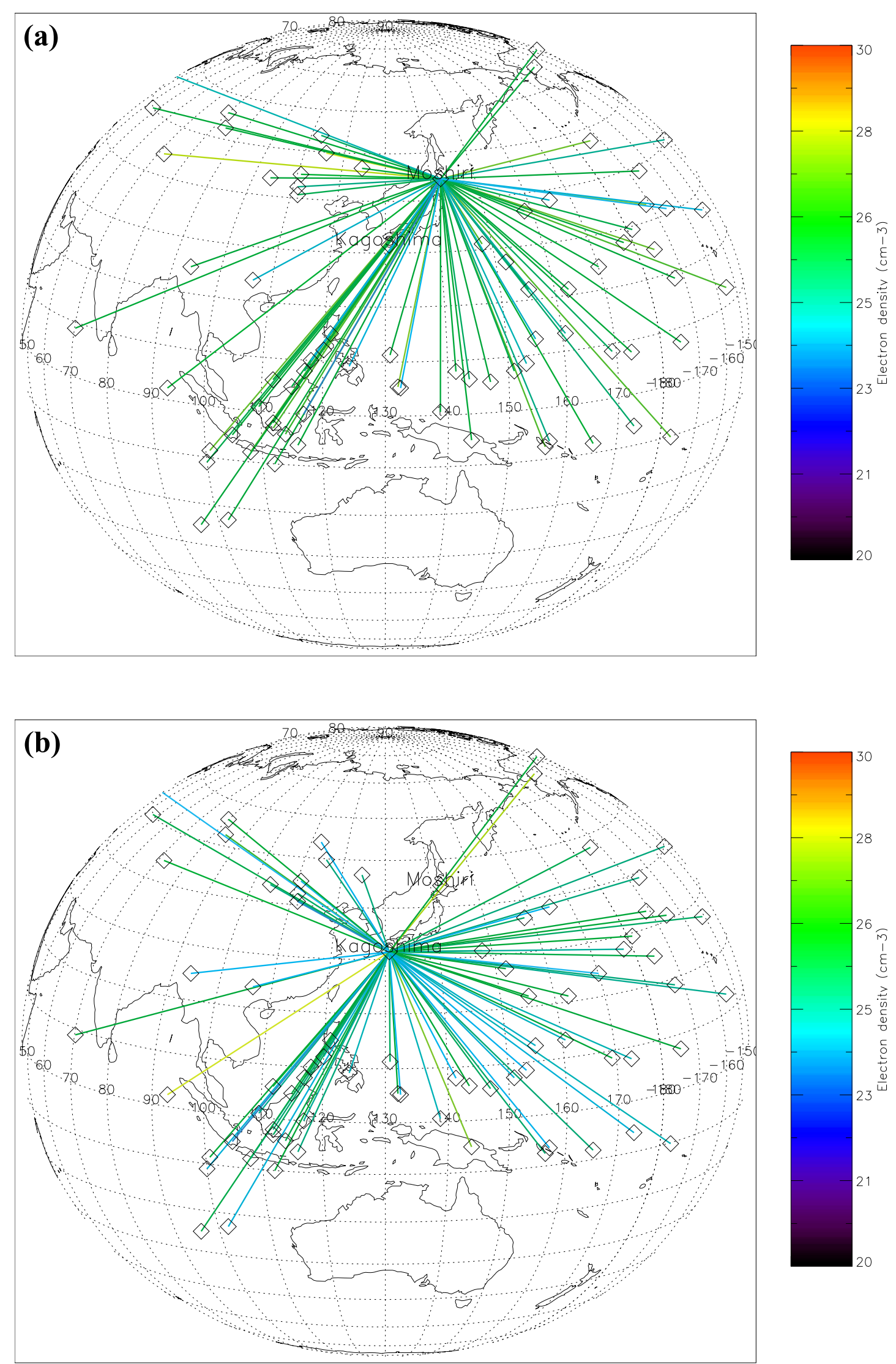

Fig. 3. Color-line distributions of nighttime electron densities analyzed from tweek atmospherics observed between 1450 and 1452 UT on October 2, 2000 for (a) Moshiri (upper panel) and (b) Kagoshima (lower panel). Empty squares show source positions of the tweek atmospherics, and colored lines connecting the source positions and the station represent equivalent electron densities, displayed by color codes. 


\section{Tweek Measurements and Comparisons with Other Measurements}

\subsection{Tweek measurements}

We picked pairs of strong tweek atmospherics received simultaneously between Moshiri and Kagoshima. On average, several dozen pairs of tweek atmospherics were identified per minute. Here, we examine equivalent electron densities obtained from first-order-mode cut-off frequencies and analyses of nighttime tweek atmospherics. Figure 3 shows colorline distributions of equivalent electron densities derived from tweek atmospherics' pairs identified between Moshiri and Kagoshima from 1450-1452 UT (LT = UT +9 h) on October 2, 2000. Figure 3(a) shows the distribution for Moshiri, and (b) shows the distribution for Kagoshima. The empty squares show the source positions of the tweek atmospherics. Source positions were fixed by crossing the propagation distances from Moshiri and Kagoshima. The colored lines between the source positions and the respective station represent equivalent electron densities calculated along the propagation path, which are displayed by color codes. We found that the tweek sources were distributed in a wide area surrounding the Japanese islands, and the electron densities ranged from $20-28 \mathrm{el} . / \mathrm{cm}^{3}$, which corresponded to reflection heights of $80-85 \mathrm{~km}$. We also noticed that the distribution for Moshiri was almost uniform, with higher densities (24-28 el. $\left./ \mathrm{cm}^{3}\right)$ over a wide area. The distribution for Kagoshima, however, was not uniform, and showed lower densities (23-25 el. $\left./ \mathrm{cm}^{3}\right)$ in some directions, particularly in the southeastern direction.

The source positions of the tweek atmospherics shown in Fig. 3 were confirmed by lightning source data collected by the Lightning Imaging Sensor (LIS) on the TRMM satellite. Figure 4 shows the distribution of lightning sources in East Asia, Australia, and the Pacific on October 2, 2000, as obtained by the LIS. Available LIS search areas are limited to latitudes between $40^{\circ} \mathrm{S}$ and $40^{\circ} \mathrm{N}$. The sensor showed active lightning sources in the southern part of China and Indochina. However, as shown in Fig. 3, only a few tweek sources were fixed there. Fixing sources may be difficult along the baseline area between two stations (Nishino and Kashiwagi, 1976). The LIS data and tweek estimations show roughly consistent source distributions in the area from Indonesia to the Pacific Ocean. Figure 3 shows some tweek sources in northern and northwestern China. However, these areas are out of the LIS range.

Below, we compare the equivalent electron densities estimated by the tweek atmospherics to the electron density profiles obtained by the IRI-95 model, MF radar, and rocket experiments.

\subsection{Comparison with the IRI-95 model}

The electron density distribution obtained by the IRI-95 model at $\sim 1500 \mathrm{~h} \mathrm{UT}(0000 \mathrm{~h} \mathrm{LT})$ on October 2 was nearly uniform along the meridian planes in the low-middle latitudes (not shown here). In Fig. 5, we show electron density profiles at longitudes of $70^{\circ} \mathrm{E}, 130^{\circ} \mathrm{E}$, and $190^{\circ} \mathrm{E}$ and $10^{\circ} \mathrm{N}$ latitude, where many tweek sources were fixed. The local time at $70^{\circ} \mathrm{E}$ and $190^{\circ} \mathrm{E}$ corresponds to $2000 \mathrm{~h} \mathrm{LT}$ and $0400 \mathrm{~h} \mathrm{LT}$, respectively, which is close to daytime. We found that the electron densities of $20-28 \mathrm{el} . / \mathrm{cm}^{3}$ estimated by the tweek method coincided with those determined by the
IRI-95 model for $80-82 \mathrm{~km}$ heights. This indicates that the tweek method is useful for estimating electron densities in the lower part of the D-region ionosphere. Model electron densities at $80-82 \mathrm{~km}$ heights were higher at $70^{\circ} \mathrm{E}$ (evening) and $190^{\circ} \mathrm{E}$ (morning) than at $130^{\circ} \mathrm{E}$ (nighttime). The measured electron densities at Kagoshima showed a non-uniform distribution, as shown in Fig. 3(b). The distribution had lower electron densities in the southeastern direction (nighttime) nearer to Kagoshima than in the eastern/western directions (morning/evening) farther from Kagoshima. This aspect seems consistent with the IRI-model, but it was less exact. At shorter propagation distances, the method may be less reliable for tracking the dispersion of tweeks. Further studies of tweek atmospherics over different days are needed.

\subsection{Comparison with MF radar data}

MF radar is a useful tool for measuring electron densities in the lower ionosphere. Ionospheric absorption of MF radio waves differs between the O-mode and the X-mode. The difference (ratio) between the echo intensities of the two modes is a function of electron densities in the lower ionosphere and is named after the Differential Absorption Experiment (DAE). To check the equivalent electron densities obtained by the tweek method, we examined electron density profiles measured by MF radars at Wakkanai $\left(45.36^{\circ} \mathrm{N}, 141.81^{\circ} \mathrm{E}\right)$ and Yamagawa $\left(31.20^{\circ} \mathrm{N}, 130.62^{\circ} \mathrm{E}\right)$, which are located near Moshiri and Kagoshima, respectively (Fig. 2). The MF radar has a 2-km step height resolution for measuring electron density (Igarashi et al., 2000).

Figure 6 shows monthly mean electron density profiles at 14:20-15:20 UT at Wakkanai and Yamagawa. These monthly profiles were taken on geomagnetically quiet days during October 2000, for comparison with the quiet-day electron densities on October 2 obtained by the tweek method. The electron density profile at $130^{\circ} \mathrm{E}$ by the IRI95 model (Fig. 5) is also included. Holdsworth et al. (2002) revealed that nighttime electron density profiles measured by MF radar are reliable at altitudes above $84 \mathrm{~km}$, but are less reliable below $84 \mathrm{~km}$ owing to the larger O-mode noise level (however, this trend may be site specific). Here, we compare the electron densities at altitudes of $84-85 \mathrm{~km}$ as measured by MF radar with those estimated by the tweek method. The mean electron densities found by both MF radars were 70$130 \mathrm{el} . / \mathrm{cm}^{3}$ at $84 \mathrm{~km}$ altitude, which is higher by about a factor of five than the densities determined by the tweek method. Electron densities at Wakkanai were also higher than the densities determined at Yamagawa, which is consistent with the distributions obtained by the tweek method, as shown in Fig. 3. Note that the electron density profile at Wakkanai and the IRI-95 model show similar profiles between altitudes of $84 \mathrm{~km}$ and $92 \mathrm{~km}$. If we extrapolate the profile at Wakkanai to the lower-altitude part of the IRI-95 profile, electron densities can be estimated as $20-28 \mathrm{el} . / \mathrm{cm}^{3}$ at a height of $82 \mathrm{~km}$. This result indicates that electron densities estimated by the tweek method may be consistent with the densities determined by MF radar at the lower part of the D-region ionosphere.

\subsection{Comparison with rocket data}

Direct measurements by rockets can provide the most reliable electron density data in the D-region ionosphere. Rocket experiments in the low latitudes have been carried 


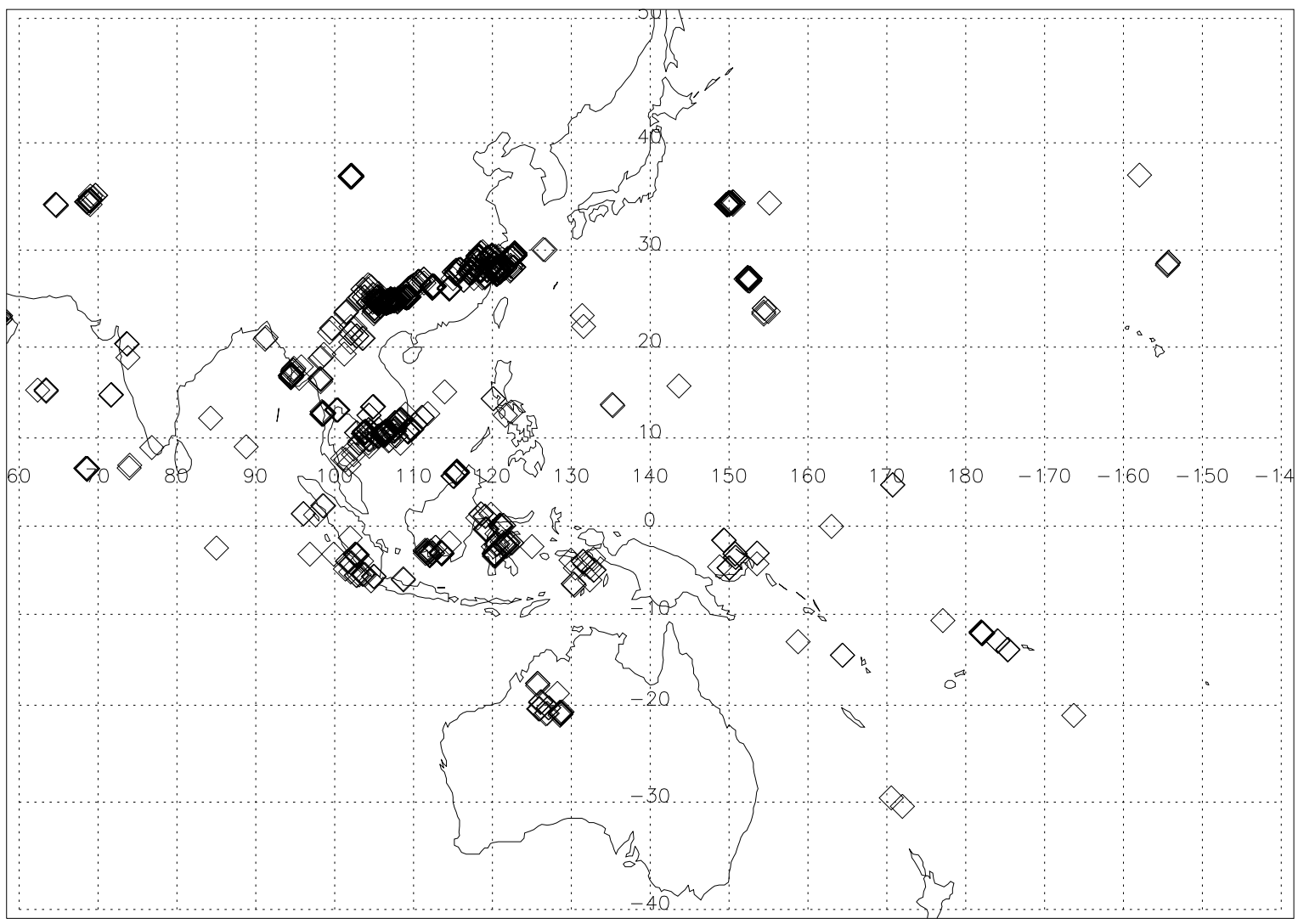

Fig. 4. Distribution of lightning sources in the East Asia-Pacific region on October 2, as obtained by the Lightning Imaging Sensor (LIS) on board the TRMM satellite.

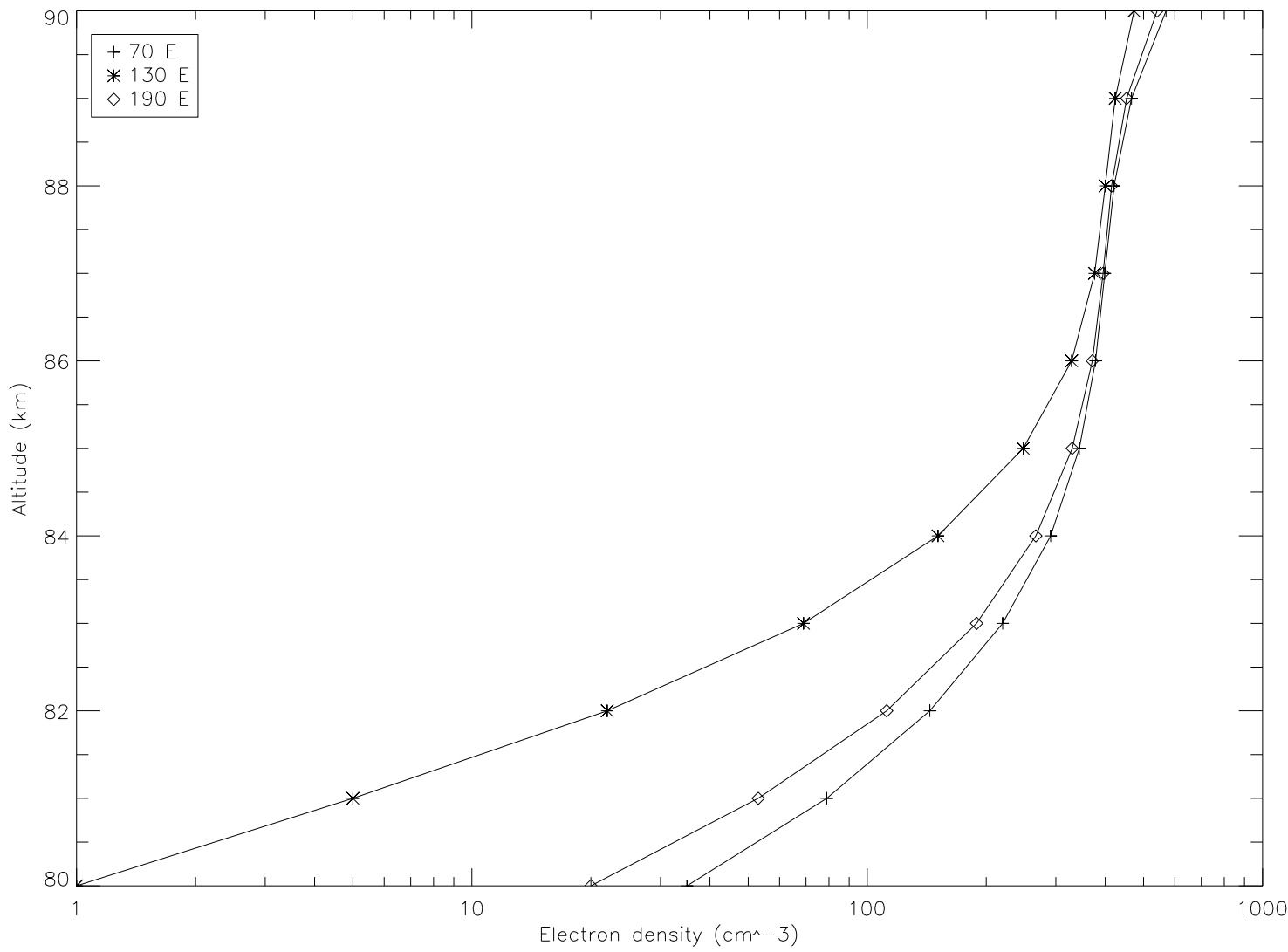

Fig. 5. Electron density profiles at longitudes of $70^{\circ} \mathrm{E}, 130^{\circ} \mathrm{E}$, and $190^{\circ} \mathrm{E}$ and latitude $10^{\circ} \mathrm{N}$ obtained by the IRI- 95 model. 


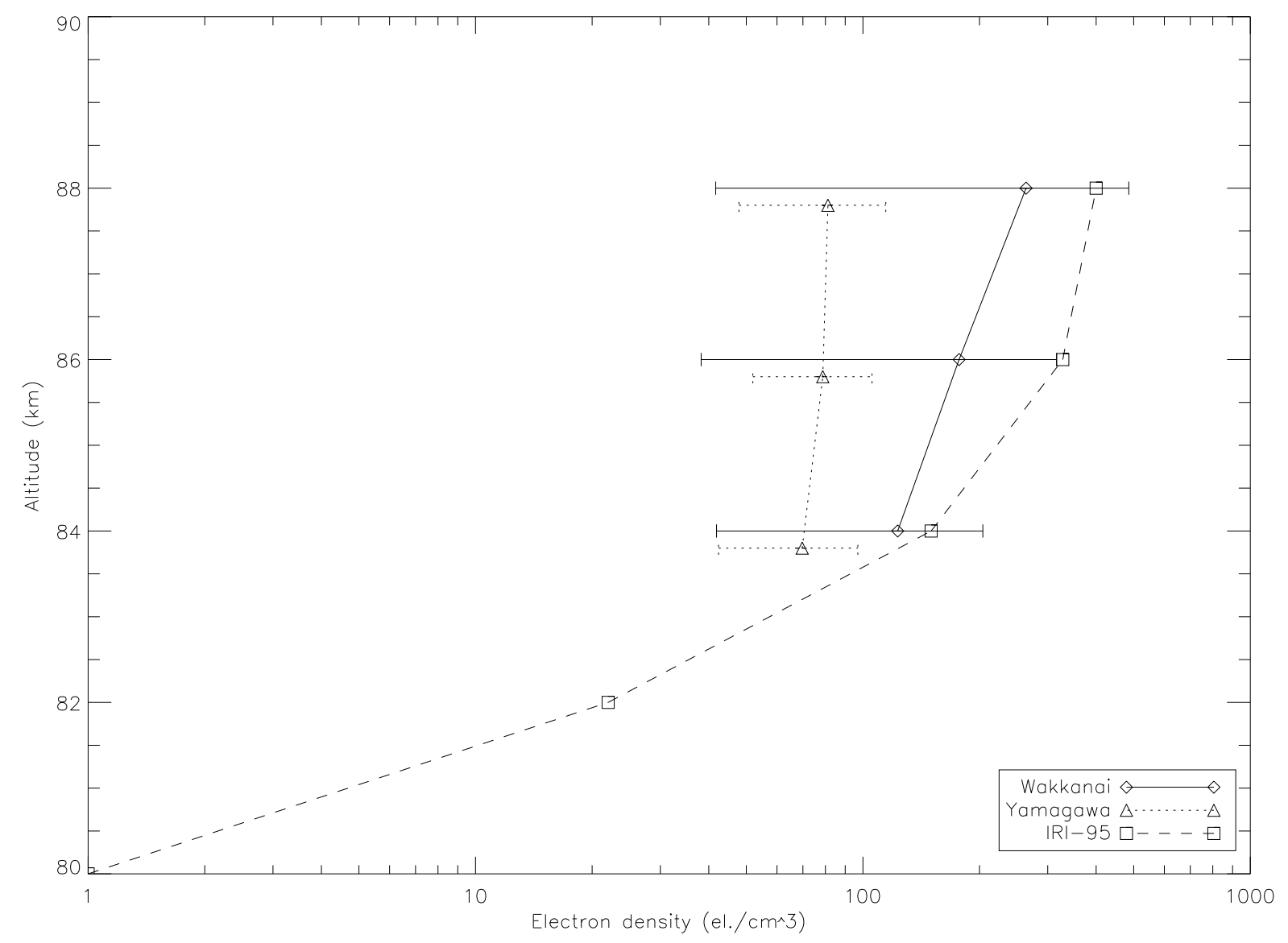

Fig. 6. Monthly mean electron density profiles for 14:20-15:20 UT measured by MF radar at Wakkanai and Yamagawa. The electron densities were averaged from profiles taken on geomagnetically quiet days in October 2000. Horizontal bars represent standard deviations from the mean values. The electron density profile by the IRI-95 model is also shown.

out many times under various solar-activity and geophysical conditions. Among these experiments, we chose electron density data collected during high solar activity to match tweek measurement conditions in October 2000. Nighttime rocket measurements recorded between 1961 and 1968 in Japan showed average electron densities of 30-400 el. $/ \mathrm{cm}^{3}$ at altitudes of 80-90 km, and electron densities varied sharply between 20 and $50 \mathrm{el} . / \mathrm{cm}^{3}$ in the lower part (80-84 km) of the D-region ionosphere (Maeda, 1971). Other nighttime rocket measurements in Japan showed electron densities of $10-1000 \mathrm{el} . / \mathrm{cm}^{3}$ at altitudes of $83-90 \mathrm{~km}$ during periods of high solar-activity, with electron densities varying sharply between 10 and $100 \mathrm{el} . / \mathrm{cm}^{3}$ in the lower part (83-85 km) of the D-region ionosphere (Nagano and Okada, 2000). These findings confirm that the equivalent electron densities determined by the tweek method agree with rocket-experiment measurements of electron densities in the lower part of the D-region.

\section{Conclusions}

We estimated electron densities in the low-middle latitude D-region ionosphere using the frequency dispersion characteristics of tweek atmospherics. This method uses accurate readings of the first-order mode cut-off frequency determined by least-mean-square method calculations. Estimated electron densities were equivalent to the reflection height of the first-mode propagation waves of tweeks. The equiva- lent electron densities ranged from $20-28 \mathrm{el} . / \mathrm{cm}^{3}$ at reflection heights of $80-85 \mathrm{~km}$ during the night of October 2 , 2000. Comparing tweek method results with electron density profiles from the IRI-95 model, MF radar, and previous rocket experiments, we found almost consistent values for estimated and measured electron densities in the lower part of the D-region ionosphere. The tweek method has the unique advantage of being capable of estimating electron densities (reflection heights) in a wide area surrounding Japan. This suggests that the tweek method could be used for detecting changes in reflection heights in the D-region ionosphere, which could correspond to abnormal geophysical conditions such as geomagnetic storms.

Acknowledgments. We wish to thank Y. Ikegami at the Moshiri Observatory and K. Hidaka at the Kagoshima Observatory, STE Laboratory, Nagoya University for supporting the ELF/VLF wave observations. Electron densities by the IRI-95 model and LIS data were obtained from the web pages of the National Space Science Data Center, and the Global Hydrology and Climate Center, NASA, respectively.

\section{References}

Bickel, J. E., J. A. Ferguson, and G. V. Stanley, Experimental observation of magnetic field effects on VLF propagation at night, Radio Science, 5, 19, 1970.

Budden, K. G., Radio Waves in the Ionosphere, Cambridge University Press, 1961.

Hayakawa, M., K. Ohta, and K. Baba, Wave characteristics of tweek atmo- 
spherics deduced from the direction-finding measurement and theoretical interpretation, J. Geophys. Res., 99, 10733-10743, 1994.

Holdsworth, D. A., R. Vuthaluru, I. M. Reid, and R. A. Vincent, Differential absorption measurements of mesospheric and lower thermospheric electron densities using the Buckland Park MF radar, J. Atmos. Sol.-Terr. Phys., 64, 2029-2042, 2002.

Igarashi, K., Y. Murayama, M. Nagayama, and S. Kawana, D-region electron density measurements by MF radar in the middle and high latitudes, Adv. Space Res., 25, 25-32, 2000.

Maeda, K-i., Study on electron density profile in the lower ionosphere, $J$. Geomag. Geoelectr., 23, 133-159, 1971.

Nagano, I. and T. Okada, Electron density profiles in the ionospheric Dregion estimated from MF radio wave absorption, Adv. Space Res., 25, 33-42, 2000.

Nishino, M. and M. Kashiwagi, The characteristics of the distribution of atmospherics and the consideration of the fixing error, Proc. Res. Inst. Atmos., Nagoya University, 23, 1-16, 1976.

Outsu, J., Numerical study of tweeks based on waveguide mode theory, Proc. Res. Inst. Atmos., Nagoya University, 7, 58-71, 1960.

Ryabov, B. S., Tweek propagation peculiarities in the Earth-ionosphere waveguide and low ionosphere parameters, Adv. Space Res., 12(6), (6)255-(6)258, 1992.

Shimakura, S., M. Moriizumi, and M. Hayakawa, On the observation of the ionospheric disturbances based on dispersion property of atmospher- ics propagation in the Earth-ionosphere waveguide, The IEICE Transactions on Communications (Japanese Edition), A·P90-17, 27-33, 1990 (in Japanese).

Shvets, A. V. and M. Hayakawa, Polarization effects for tweek propagation, J. Atmos. Sol.-Terr. Phys., 60(4), 461-469, 1998.

Thomas, L. and M. D. Harrison, The electron density distributions in the D-region during the night and pre-sunrise period, J. Atmos. Terr. Phys., 55, 173, 1970 .

Thomson, N. R., Experimental daytime VLF ionospheric parameters, $J$. Atmos. Terr. Phys., 55, 173, 1993.

Wait, J. R., Electromagnetic Waves in Stratified Media, Pergamon Press, $147,1970$.

Yamashita, M., Propagation of tweek atmospherics, J. Atmos. Terr. Phys., 40, 151, 1978.

Yano, S., T. Ogawa, and H. Hagino, Waveform analysis of tweek atmospherics, Research Letter of Atmosheric Electricity, 9, 31-42, 1989.

Yedemsky, D., B. S. Ryabov, A. Shchokotov, and V. S. Yarotsky, Experimental investigation of the tweek field structure, Adv. Space Res., 12, 251-254, 1992.

H. Ohya (e-mail: ohya@faculty.chiba-u.jp), M. Nishino, Y. Murayama, and K. Igarashi 